Canadian University Music Review

Canadian University Music Review

Revue de musique des universités canadiennes

\title{
Répertoire des travaux universitaires sur la musique du Québec (1924-1984)
}

\section{Marie-Thérèse Lefebvre}

Numéro 6, 1985

URI : https://id.erudit.org/iderudit/1014032ar

DOI : https://doi.org/10.7202/1014032ar

Aller au sommaire du numéro

Éditeur(s)

Canadian University Music Society / Société de musique des universités canadiennes

ISSN

0710-0353 (imprimé)

2291-2436 (numérique)

Découvrir la revue

Citer ce document

Lefebvre, M.-T. (1985). Répertoire des travaux universitaires sur la musique du Québec (1924-1984). Canadian University Music Review / Revue de musique des universités canadiennes, (6), 45-57. https://doi.org/10.7202/1014032ar

(C) Canadian University Music Society / Société de musique des universités canadiennes, 1985
Ce document est protégé par la loi sur le droit d'auteur. L'utilisation des services d’Érudit (y compris la reproduction) est assujettie à sa politique d'utilisation que vous pouvez consulter en ligne.

https://apropos.erudit.org/fr/usagers/politique-dutilisation/ 


\title{
RÉPERTOIRE DES TRAVAUX UNIVERSITAIRES SUR LA MUSIQUE DU QUÉBEC (1924-1984)
}

\author{
Marie-Thérèse Lefebvre
}

Ce premier inventaire des travaux universitaires traitant de la musique québécoise a pour but de présenter l'état de la recherche sur notre vie musicale et de faire connaître les travaux en cours. Il enregistre les travaux dirigés, mémoires de maîtrise et thèses de doctorat déposés dans les universités canadiennes et étrangères. Pour réaliser ce projet, nous avons consulté les bibliothèques des universités suivantes: Montréal, McGill, Concordia, Université du Québec à Montréal, Laval et Western, ${ }^{1}$ et celles des conservatoires de Montréal et de Québec. Nous avons aussi consulté les volumes suivants:

ADKINS, C., DICKINSON, A. éds.

1977: International Index of Dissertations and Musicological Works in Progress. Philadelphia: American Musicological Society: International Musicological Society, first edition.

BRADLEY, I.A.

1976: Selected Bibliography of Musical Canadiana. Agincourt: GLC Publishers Limited, revised edition.

BRISSON, I.

1983: "Répertoire des membres de l'Association pour l'avancement de la recherche en musique du Québec ", Cahiers de l'ARMuQ, No. 1.

CHARTIER, Y.

1979: "Situation de la recherche sur la musique au Canada français ", Bulletin du centre de recherche en civilisation canadienne-française, Université d'Ottawa, No. 19, 1-14.

GRIBENSKI, J.

1979: Thèse de doctorat en langue française relatives à la musique: bibliographie commentée. New York: Pendragon Press. 
HALL, F.A., et al.

1970: Basic Bibliography of Musical Canadiana (mimeographed). Toronto: University of Toronto, Faculty of Music Library. POTVIN, G., KALLMANN, H., WINTERS, K.

1983: Encyclopédie de la musique au Canada. Montréal: Fides. SANDVOSS, J.

Canadian Graduate Theses in Music and Music Education (copie dactylographiée non datée, déposée au centre de documentation de l'Encyclopédie de la musique au Canada localisé à la faculté de musique de l'Université de Montréal).

Nous présentons ce répertoire par ordre chronologique pour mettre en évidence, d'une part, le caractère historique de ces recherches et la variation des sujets selon les époques et d'autre part, pour démontrer la vitalité actuelle de la recherche par le nombre croissant des sujets. Nous y avons aussi ajouté les thèses en composition, car chacune des oeuvres est accompagnée d'une analyse du compositeur ainsi que celles en ethno-musicologie lorsqu'elles étudient la musique des Inuit, des Indiens ou de notre folklore. Enfin, ce travail nous a permis de faire une mise à jour des sujets de thèse répertoriés dans les diverses bibliographies, certains d'entre eux ayant été abandonnés ou ayant subi des modifications importantes.

Nous souhaitons, par ce premier recueil, inviter les étudiants et les chercheurs indépendants (en dehors du réseau universitaire) faisant actuellement des recherches en vue de publication, à inscrire le titre de leur travail au Centre québécois de dépôt des sujets de recherche en musique (projet pilote mis de l'avant par le conseil de l'Association pour l'avancement de la recherche en musique du Québec). ${ }^{2}$ Nous aurons alors une image précise et complète de la recherche sur la musique du Québec.

Nous remercions Claude Beaudry, Juliette Bourassa, Irène Brisson, George A. Proctor, Claire Versailles et Stephen Willis pour leur précieuse collaboration. ${ }^{3}$ 


\section{NOTES}

1. Sous la direction de Monsieur G.A. Proctor, la faculté de musique de l'Université Western a mis sur ordinateur toutes les thèses sur la musique canadienne jusqu'en 1979.

2. ARMuQ - Dépôt des sujets C.P. 695, Tour de la Bourse, Montréal, Québec, H4Z 1J9.

3. Nous indiquons le titre du diplôme, le département ou la faculté lorsque l'information est donnée, et l'université qui a décerné le titre. Les travaux dirigés produits à la faculté de musique de l'Université de Montréal ne sont pas disponibles à la bibliothèque. Il faut communiquer avec l'auteur de la recherche.

1924 : MATHEWSON, D.R.

French Canadian Folk Songs M.A., McGill University. 1932 : PELLETIER, J.R.

A perçu historique sur le chant liturgique de l'Église en Europe et dans la province de Québec. Doctorat, Université Laval.

1934 : MONTREUIL, A.

[Le chant grégorien] D. Mus., Université Laval (la page titre manque).

1942 : CARDIN, C.

Bio-bibliographie de Marius Barbeau. Mémoire, École de Bibliothéconomie, Université de Montréal.

1943 : LAPERRIERE, L.

Bio-bibliographie de Joseph Quesnel. Mémoire, École de bibliothéconomie, Université de Montréal.

1947 : ROGERS, R.A.

French Canadian Folk Music. M.A., Northwestern University.

1948 : PIERRE-ALPHONSE, Frère

Chant et musique sacrée de la nouvelle France. M.A., Histoire, Université d'Ottawa.

1950 : LAFLAMME, C.

Bibliographie analytique de la littérature musicale canadienne française. Mémoire, École de Bibliothéconomie, Université Laval.

POUINARD, A.A.

Recherches sur la musique d'origine française en Amérique du Nord: Canada et Louisiane. Doctorat, Lettres, Université Laval.

1951 : MARIE URSULE, Sr.

La civilisation traditionnelle des Lavalois. Ph. D., Université Laval. 
ROUSSEAU, M.

1955 : DEMERS, C.

The Rise of Music in Canada. M.A., Columbia University.

Musique et théâtre à Québec, 1764-1800. Licence, Histoire, Université Laval.

1956 : AMTMANN, W.

La vie musicale en Nouvelle France. Ph. D., Strasbourg. CLOUTIER, J.

La part de la musique dans le loisir de quatre-vingt six étudiants. M.A., Musique, Université de Montréal. YOUNG, R.S.

Vieilles chansons de Nouvelle France. Ph. D., Université Laval.

1959 : PREY, C.

Formation et métamorphose d'une chanson: le canard blanc.

D.E.S. Lettres, Université Laval.

1961 : POULIN, R.

L'oeuvre vocale de Jean Papineau-Couture. Licence, Musique,

Université de Montréal.

1962 : ALLARD, G.

La technique pianistique de Paul Loyonnet. Licence, Musique, Université de Montréal.

1963 : TRAVERSY, P.

Bio-bibliographie de l'oeuvre musicale du révérend frère Barnabé. Mémoire, École de Bibliothéconomie, Université Laval.

1964 : GERMAIN, P.

La musique d'orgue au Canada: essai de bibliographie sélective (1949-1963). Mémoire, École de bibliothéconomie, Université d'Ottawa.

MARIE PAULINE DE L'ASSOMPTION

Évaluation de la situation de la musique liturgique à Montréal. Licence, Musique, Université de Montréal.

1965 : ROUSSEAU, N.

La Chanson canadienne et les jeunes. M.A. Sciences religieuses, Université d'Ottawa.

VEZINA, $R$.

La musique au Petit Séminaire de Québec: la Société SainteCécile. Licence, Histoire, Université Laval.

1966 : BEGIN, C.

La vie musicale sous le régime français. Licence, Musique, Université de Montréal.

1968 : GUIMOND, P.

La chanson comme phénomène socio-culturel: analyse de ses divers aspects. M.A., Musique, Université de Montréal. 
O'NEILL, M.E.

A plan for the development of a Curriculum in Music for Marianapolis College, Montreal. D. Ed., Department of Education, Columbia University.

ROY, M.

L'histoire de la musique de l'Institut des Soeurs de la Charité de Québec. M.A., Université Laval.

THIBEAULT, L.M.

The Complainte in French Canadian Folk Music. M.A., University of Washington.

1969 : ABBOTT, E.O.

The Evolution of the Canadian Music Festival Movement as an Instrument of Education. Ph. D., Boston University. HIRBOUR, L.

Traduction de l'article de $\mathrm{H}$. Kallmann " Historical Background " in: Aspects de la musique au Canada, M. Kendergi et G. Potvin éds., Montréal, Centre de Psychologie et de Pédagogie, 1970. Licence, Musique, Université de Montréal. OWEN, S.

Piano Concerto in Canada since 1955. Ph. D., University of Washington.

RICHER, L.

Mémoire présenté à la Commission Deschênes. Licence, Musique, Université de Montréal.

1970 : BEAUBIEN, M.L.

Survey of Keyboard Music of Canadian Composers since 1900. M.A., Indiana University.

GAUTHIER-OUELLET, Y.

McLaren et le son animé. Licence, Musique, Université de Montréal.

LAFORTE, C.,

La classification de la chanson folklorique française d'après

les poétiques de tradition orale. D.E.S., Université Laval.

LISTER, W.W.,

The Contemporary Sonata for Violin and Piano by Canadian Composers. D.M.A., Boston University.

PILOTE, G.

L'enseignement du solfège dans les écoles élémentaires de la Commission des Écoles Catholiques de Montréal: Claude Champagne et ses contributions. M.A., Musique, Université de Montréal.

PROVOST, M.P.

Claude Champagne, l'un des nôtres. Licence, Musique, Université de Montréal.

1971 : GRENIER, A.

Sonate pour deux pianos de Bruce Mather. M.A., Musique, Université de Montréal. 
MALOUIN-GELINAS, F.

La vie musicale dans la ville de Québec de 1840-1845. M.A., Musique, Université de Montréal.

PERREAULT, J.G.

Une maîtrise: les petits chanteurs du Mont Royal. M.A., Musique, Université de Montréal.

1972 : BAIL-MILOT, L.

L'oeuvre et les procédés de composition chez Claude Champagne. M.A., Université de la Sorbonne, Paris.

BOURASSA-TREPANIER, J.

Rodolphe Mathieu (1890-1962). Doctorat, Université Laval. CLICHE, R.

La musique de piano pour les jeunes. Ph. D., Université Laval. DENIS, C.

Cérébralisme et lyrisme dans l'oeuvre de J.P. Couture. M.A., Musique, Université de Montréal.

LAPLANTE, L.

Essai de systématisation de chants amérindiens. M.A., Musique, Université de Montréal.

WALSH, A.

The Life and Works of Claude A. Champagne. Ph. D., Catholic University of America.

1973 : LEFEBVRE-BOUVRY, N.

Analyse du système actuel de l'enseignement musical au secondaire. M.A., Musique, Université de Montréal.

CHAPMAN, N.

Piano Music by Canadian Composers (1940-1965). Ph. D., Case Western Reserve University.

EVANGELISTA, J.

Dances (commentaires). M. Mus., Musique, Université de Montréal.

JODOIN-PILON, M.T.

Réflexions sur une initiation à la musique contemporaine à l'école secondaire. M.A., Musique, Université de Montréal.

MAUGER, L.

L'évaluation du système d'enseignement musical à l'élémentaire dans la région de Montréal (1972-73). M.A., Musique, Université de Montréal.

1974 : CROUSSET, D.

L'orgue de la basilique de l'Oratoire St-Joseph. M.A., Musique, Université de Montréal.

FOUILLANT, L.

Jeux et Glissements, pièce circulaire pour orgue (Genèse et analyse). M.A., Musique, Université de Montréal. 
1975 : GAGNÉ, R.

Un siècle d'activités musicales à St-Rock de Québec. Conservatoire de musique de Québec, concours d'histoire de la musique et musicologie, premier prix 1975.

LONGTIN, M.

Le pélerin d'Alneoil, pour orchestre, Copie conforme, musique de film. M.A., Musique, Université de Montréal.

PAGE, T.

L'enseignement de la musique vocale et instrumentale aux Ursulines de Québec de 1830 à 1880 (2 vol.). M.A., Musique, Université Laval.

ROBERGE, J.

Gilles Tremblay, sa vie, son oeuvre. Conservatoire de musique de Québec, concours d'histoire de la musique et musicologie, mention 1975.

SLEMON, P.

Montreal's Musical Life under the Union with an Emphasis on the Terminal Years (1841-1867). M.A., Faculty of Music, McGill University.

TROCHU, P.

Eros (oeuvre électro-acoustique), Micrarose, pour 12 musiciens. M.A., Musique, Université de Montréal.

VILLENEUVE, C.

Léo Pol Morin, musicographe. M.A., Musique, Université de Montréal.

1976 : GOUIN, J.

Débat, Distorsion. M.A., Musique, Université de Montréal. ROY, M.

Spyx: la mort était extravagante, TSE TNANT: Te Deum. M.A., Musique, Université de Montréal.

LEFEBVRE, M.C.

Radiance de François Morel. M.A., Musique, Université de Montréal.

POIRIER-BLANCHARD, C.

L'organisation de la musique symphonique à Québec de 19351942: dissension et fusion. Mémoire de Baccalauréat, Université Laval.

TURBIDE, N.

Charles Albert Edwin Harriss: the McGill Years. M.A., Faculty of Music, McGill University.

1977 : DESROCHES, M.

Ce qui s'est écrit sur la musique traditionnelle des Inuit: un bilan. M.A., Musique, Université de Montréal.

LAFORTE, C.

Survivance de la laisse dans la chanson de tradition orale.

Doctorat, Université Laval. 
MARINEAU, P.B.

L'église paroissiale de Québec - Son service musical, (17601865). Conservatoire de musique de Québec, concours 1978 : HARVEY, D. d'histoire de la musique et musicologie, deuxième prix 1977. Analyse stylistique et comparative des jeux de gorge du Nordest canadien. M.A., Musique, Université de Montréal.

WALTZ, M.J.

Cinq madrigaux de Bruce Mather: une analyse formelle et stylistique. M.A., University of Western Ontario.

1979 : BEGIN, C.

La musique traditionnelle pour violon: Jean Carignan. Doctorat, Musique, Université de Montréal.

BELAND, $\mathrm{M}$.

La vie des voyageurs et des forestiers par leurs chansons. M.A., Faculté des Arts, Université Laval.

BONENFANT, L.

Étude comparée de la chanson "Par derrière chez ma tante ". M.A., Lettres, Université Laval.

BOOTHROYD, D.A.

Pentland, Freedman, Prévost: Three Canadian String Quartets (1968-1972). M.A., University of Western Ontario. FRECHETTE, F.

La question de l'enseignement des rudiments de la théorie musicale au niveau secondaire I. M.A., Musique, Université Laval.

GOUGEON, D.

Berceuse. Maïté II (Ne me dérangez pas, St-Denys Garneau) Concerto Dello Spirito. M.Mus., Musique, Université de Montréal.

L'ECUYER-LACROIX, S.

Les feuilletons musicaux de Joseph d'Ortigue au Journal des débats (1852-1866). M.A., Musique, Université Laval.

MONTPETIT, C.

Musique Inuit du Québec arctique: contribution à une méthode d'analyse en anthropologie de la musique. M.A., Anthropologie, Université Laval.

SAUVE, $M$.

Époques (algorythmiques). M.Mus., Musique, Université de Montréal.

1980 : BERTHIAUME, C.

Le katajjaq, un cas de polyphonie bi-dimentionnelle. M.A., Musique, Université de Montréal.

GUILBAULT, J.

Analyse mélodique de la chanson en laisse "Trois beaux canards" (travail dirigé). M.A., Musique, Université de Montréal. 
MAGNAN, O.

La vie musicale à Québec de 1908 à 1918. Conservatoire de musique de Québec, concours de musicologie, premier prix 1980.

MESSIER, A.M.

Répertoire des jeunes compositeurs de la région de Québec (mémoire de fin baccalauréat). B.Mus., Musique, Université Laval.

QUENNEVILLE, P.

Guillaume Couture (1851-1915), animateur de la vie musicale montréalaise (d'après le fonds Couture) (travail dirigé). M.A., Musique, Université de Montréal.

DESTREMPES-VALIQUETTE, J.

Poèmes et chants de la Résistance: un langage musical populaire différent. M.A., McGill University.

FAVRETTI, C.

Inventaire de la revue Passe-Temps à partir du fonds Villeneuve (travail dirigé). M.A., Musique, Université de Montréal.

GAGNÉ, M.

Les sources d'inspiration extra-musicales de quelques compositeurs québécois (1945-1975). M.A., Musique, Université de Montréal.

LACOMBE, A.

En phase illimitée, Parcours 72, Huis Clos. M.Mus., Musique, Université de Montréal.

LEFEBVRE, M.-T.

Nouvelle approche du matériau sonore dans les oeuvres post sérielles: analyse du Quintette de Garant. Ph. D., Musique, Université de Montréal.

1982 : BELAND, $M$.

Anthologie de chansons des voyageurs, forestiers et coureurs des bois. M.A., Université Laval.

BOUDREAU, $M$.

Croissances, Croisées, Phases métronomiques. M.Mus., Musique, Université de Montréal.

BRULOT-KHELIFA, $M$.

Inventaire de la revue Passe-Temps à partir du fonds Villeneuve (travail dirigé). M.A., Musique, Université de Montréal.

DESCHENES, D.

Le répertoire d'une chanteuse gaspésienne: son interprétation, l'analyse du comportement rythmique et des structures musicales. M.A. Histoire, Université Laval. 
FRENETTE, C.

La notion d'hétérophonie dans la musique des jeunes compositeurs québécois (travail dirigé). M.Mus., Musique, Université de Montréal.

LAUBERT, A.

Analyse du geste créateur (travail dirigé). Osmose, pièce symphonique no. 2. Valse concertante, piano et orchestre. M.Mus., Musique, Université de Montréal.

MATTEAU, C.

Le concours international de musique de Montréal (travail dirigé). M.A., Musique, Université de Montréal.

1983 : BARRIAULT, J.

Inventaire des découpures de journaux conservées dans le fonds Wilfrid Pelletier aux Archives nationales du Québec à Montréal. M.A., Musique, Université de Montréal.

DESCHESNES, B.

Dimensions, Chant/Chrystal, Agone, Prisme. M.Mus., Musique, Université de Montréal.

DEVILLERS, J.B.

Trio à cordes, Iris. M.Mus., Musique, Université de Montréal. DUPRE, C.

Quatre compositions de musique commerciale (travail dirigé).

M.Mus., Musique, Université de Montréal.

GILBERT, R.

Évolution des styles à l'intérieur des trois pièces pour piano les plus récentes de Jean Papineau-Couture (travail dirigé). M.A., Musique, Université de Montréal.

GUILLEMETTE, P.

Blocage, pour quatuor de saxophones (travail dirigé). M.Mus., Musique, Université de Montréal.

LECLERC, C.

Le cantique au Canada français. M.A., Musique, Université de Montréal.

LONGTIN, M.

De St-Malo à Bourges par Bouffémont, pour orchestre. Le Jeu de l'inventaire, pour comédiens et quintette à vent. Migration vers l'automne, pour orchestre à cordes et deux percussions, Trilogie de la montagne, oeuvre électroacoustique. D.Mus., Musique, Université de Montréal.

METRAS, C.

Inventaire de la correspondance Wilfrid Pelletier à partir du fonds d'archives (A-L). M.A., Musique, Université de Montréal.

SCHINGH, D.

Quatuor à cordes, Scénario pour orchestre. M.Mus., Musique, Université de Montréal. 
TREMBLAY-MATTE, C.

Les instruments de musique sous le régime français et leurs fonctions (travail dirigé). M.A., Musique, Université de Montréal.

TROCHU, P.

Osmose, pour orchestre. Fusion, pour 24 instrumentistes.

D.Mus., Musique, Université de Montréal.

TRUDEAU, $\mathrm{N}$.

L'éducation musicale à l'école québécoise et à l'école française

à travers les programmes. Ph. D., Musique, Université de Montréal.

VEZINA, C.

Inventaire de la correspondance Wilfrid Pelletier à partir du fonds d'archives (M-Z). M.A., Musique, Université de Montréal.

1984 : EVANGELISTA, J.

Motionless Move, version pour 14 musiciens et version pour orchestre. D.Mus., Musique, Université McGill.

FRENETTE, C.

Les Etrivières, opéra en huit tableaux. Le hasard éliminé. Ici, ailleurs, la lumière. M.Mus., Musique, Université de Montréal.

1985 : GUILLEMETTE, P.

Arthur Romano, musicien et pédagogue (travail dirigé). M.A., Musique, Université de Montréal.

TETREAULT, $M$.

AL-UN, Fuzei, Tisser des spectres, Tayori (oeuvres électroacoustiques). M.Mus., Musique, Université de Montréal.

TOUSIGNANT, F.

Durées, pour orchestre, Sonate pour clavecin, Histoire, pour clarinette et piano. D.Mus., Musique, Université de Montréal.

\section{En cours:}

BARRIĖRE, $M$.

Le théâtre lyrique à Montréal et à Québec (1840-1913). Doctorat, Histoire, Université Laval.

BERDUGO, D.

Pratique de la musique liturgique et para-liturgique des Juifs originaires du Maroc à Montréal. Ph.D., Musique, Université de Montréal.

BERGERON, A.M.

Les feuilles de musique: un témoignage de l'histoire des années 1900 à 1929 au Canada. M.A., Musique, Université de Montréal. 
CHAYER-POITRAS, M.

Inventaire du fonds Pierre Mercure. M.A., Musique, Université de Montréal.

CLOUTIER, D.

Le Te Deum en Nouvelle-France à partir des Relations des Jésuites. M.A., Musique, Université de Montréal.

CLOUTIER, L.

Maurice Blackburn, compositeur de film musique: le renouvellement passe par la recherche sur le matériau. Ph.D., Musique, Université de Montréal.

COOPER, D.

A Conspectus of Operatic Performance in Canada. Ph.D., Music, Université of Toronto.

DESNOYERS, $M$.

Inventaire des programmes de concerts conservés dans le fonds d'archives Wilfrid Pelletier. M.A., Musique, Université de Montréal.

GALLAT-MORIN, E.

Un manuscrit inédit de musique d'orgue à Montréal au XVIIIe siècle. Ph.D., Musique, Université de Montréal.

GARCEAU, $\mathrm{H}$.

Essor et métamorphose de la vie musicale québécoise tels que révélés par la presse de 1856 à 1866. Doctorat, Musique, Université Laval.

GRATTON, J.

La musique nationaliste au Canada français de 1837 à 1867: reflet d'une pensée et d'une action culturelle et politique. M.A., Musique, Université de Montréal.

GRÉGOIRE-REID, C.

Les manuels de théorie musicale imprimés au Québec entre 1811 et 1911. M.A., Musique, Université Laval.

JOYAL, J.P.

"Democratic Rage Hornpipe ". Métamorphose d'un air de violon en milieu canadien-français (travail dirigé).

Portrait de la musique instrumentale traditionnelle du Québec (travail dirigé). M.A., Musique, Université de Montréal.

JUSTE-CONSTANT, V.

La musique populaire haïtienne à Montréal. M.A., Musique, Université de Montréal.

LAFOREST, A.

Les soixante mélodies sur des poèmes d'Émile Nelligan du compositeur québécois Léo Roy (1887-1974). M.A., Musique, Université Laval. 
MESSIER, A.M.,

Histoire et analyse de la musique western au Québec. M.A., Musique, Université de Montréal.

ORNSTEIN, L.

Musique de violon de Pitou Boudreau du Lac St-Jean. Doctorat, Université Laval.

PLAMONDON, C.

La vie musicale à Montréal de 1850 à 1900. M.A., Musique, Université de Montréal.

QUENNEVILLE, P.

Guillaume Couture et la rencontre des milieux montréalais, français et américain. Ph.D., Musique, Université de Montréal.

RHEAUME, C.

La création musicale chez les religieuses du Québec. M.A., Musique, Université de Montréal.

SPIER, $\mathrm{S}$.

The Dubois String Quartet, 1910-1938: Role in Montreal Music History. M.A., Musique, Université de Montréal.

TREMBLAY-MATTE, C.

La chanson canadienne-française et la création féminine.

Ph.D., Musique, Université de Montréal.

TURBIDE, $\mathrm{N}$.

Étude biographique d'Éva Gauthier (1885-1958): première cantatrice canadienne française d'avant-garde. Ph.D., Musique, Université de Montréal.

VEZINA-DEMERS, $M$.

Aspects de la vie musicale à Québec de 1790 à 1794 d'après la presse québécoise contemporaine. M.A., Musique, Université Laval. 